Appendix 


\section{A: Glossary}

\begin{tabular}{|l|l|}
\hline $\begin{array}{l}\text { AMCAM (Asociación de Mu- } \\
\text { nicipalidades con Alcaldes } \\
\text { Mapuche) }\end{array}$ & Association of Municipalities with Mapuche Mayors \\
\hline $\begin{array}{l}\text { ATM (Alianza Territorial Ma- } \\
\text { puche) }\end{array}$ & Territorial Mapuche Alliance \\
\hline CERD & Committee on the Elimination of Racial Discrimination \\
\hline $\begin{array}{l}\text { CIISOC (Centro de Investiga- } \\
\text { ciones de la Inclusión digitaly } \\
\text { la Sociedad de Conocimiento) }\end{array}$ & $\begin{array}{l}\text { Research Centre for Digital Inclusion and Social Knowl- } \\
\text { edge }\end{array}$ \\
\hline $\begin{array}{l}\text { CONADI (Concejo Nacional } \\
\text { de Desarrollo Indígena) }\end{array}$ & $\begin{array}{l}\text { The National Cooperation for Indigenous Development } \\
\text { in Chile }\end{array}$ \\
\hline $\begin{array}{l}\text { CME (Coordinación Mapuche } \\
\text { de Europa) }\end{array}$ & Mapuche Coordination in Europe \\
\hline $\begin{array}{l}\text { CTT (Concejo de Todas las } \\
\text { Tierras) }\end{array}$ & Council of All Territories \\
\hline $\begin{array}{l}\text { EZLN (Ejercito Zapatista de } \\
\text { Liberación Nacional) }\end{array}$ & Zapatista National Liberation Army \\
\hline $\begin{array}{l}\text { FEMAE (Federación Mapuche } \\
\text { de Estudiantes) }\end{array}$ & Mapuche Students' Federation \\
\hline $\begin{array}{l}\text { FONDECYT (Fondo Nacional } \\
\text { de Desarrollo Científicoy Tec- } \\
\text { nológico) }\end{array}$ & $\begin{array}{l}\text { The Chilean National Fund for Scientific and Technologi- } \\
\text { cal Development }\end{array}$ \\
\hline $\begin{array}{l}\text { CfbV (Gesellschaft für bedro- } \\
\text { hte Völker) }\end{array}$ & Society of Threatened People \\
\hline $\begin{array}{l}\text { ICT } \\
\text { danía) }\end{array}$ & Information and Communication Technologies \\
\hline JMM creación en La Arau- & International Defence Network for the Mapuche People \\
\hline $\begin{array}{l}\text { INDH (Instituto Nacional de } \\
\text { Derechos Humanos) }\end{array}$ & National Human Rights Institute \\
\hline ILO & International Labour Organisation \\
\hline JUPtice, Peace, and Integrity of Creation in the Araucanía \\
\hline
\end{tabular}




\begin{tabular}{|l|l|}
\hline OC (Observatorio Ciudadano) & Citizens' Observatory \\
\hline $\begin{array}{l}\text { RDT (Red por la Defensa de los } \\
\text { Territorios) }\end{array}$ & Network for the Defence of Territories \\
\hline SVD & Society of the Divine Word \\
\hline TMA & Transnational Mapuche Advocacy \\
\hline UNPO & Unrepresented Peoples' Organisation \\
\hline
\end{tabular}




\section{B: Words in Mapuzugun}

\begin{tabular}{|c|c|}
\hline Ayllarewe & an assemblage of Mapuche communities \\
\hline Az mapu & the ethical and normative framework of the Mapuche \\
\hline Jejipun & Mapuche ritual and mostly welcome ceremony \\
\hline Keyuwvn & to work amongst everyone/as a community \\
\hline Komkeyuayiñ & mutual help where everyone helps \\
\hline Koyang & $\begin{array}{l}\text { meetings or gatherings with long-lasting discussions } \\
\text { and speech acts }\end{array}$ \\
\hline Kultrun & Mapuche drum \\
\hline Lof & Mapuche community \\
\hline Lofdungu & any topic or thing that has a communal importance \\
\hline Lofkedaw & communal work \\
\hline Longko & community leader or chief \\
\hline Machi & healer or spiritual leader \\
\hline Malluntu & mutual and obligatory gift exchange \\
\hline Mapuzugun & Mapuche language \\
\hline Marichiweu & $\begin{array}{l}\text { resistance shout: for each Mapuche who falls, ten more } \\
\text { will rise }\end{array}$ \\
\hline Merken & smoked chili powder \\
\hline Mingako & collective work in the rural community \\
\hline Mizawvn & $\begin{array}{l}\text { when two (or more) people eat from the same plate as an } \\
\text { expression of enormous trust }\end{array}$ \\
\hline Ngillatun & $\begin{array}{l}\text { maybe one of the most important and highly complex rit- } \\
\text { ualised celebrations between Mapuche communities. It } \\
\text { is celebrated—at most—once a year }\end{array}$ \\
\hline Norfeleal & having a good time and living well \\
\hline Ngvtram & a family conversation \\
\hline Matetun & gathering to drink mate tea \\
\hline Metrem & a guest or visitor \\
\hline Palin & the traditional Mapuche sport \\
\hline Peñi & brother(s) \\
\hline
\end{tabular}




\begin{tabular}{|l|l|}
\hline Ragiñelwe & $\begin{array}{l}\text { literally "space of the middle" and describes "a cultural } \\
\text { agent, whose function is to mediate between the af- } \\
\text { fected parts," for example "between persons, where cer- } \\
\text { tain type of disencounter [leads] to a certain degree of } \\
\text { unbalance" (COTAM 2003, 1166, my translation) }\end{array}$ \\
\hline Rewe & family unit and ceremony site \\
\hline Trafkintu & fair and equal exchange \\
\hline Trawvn & meeting or gathering \\
\hline Trutruka & Mapuche horn \\
\hline Wallmapu & $\begin{array}{l}\text { the historical territory of the Mapuche-the southern } \\
\text { parts of today's Chile and Argentina }\end{array}$ \\
\hline Weichafe & Mapuche warrior \\
\hline Wenufoye & $\begin{array}{l}\text { the Mapuche flag accepted by most of the organisations } \\
\text { and communities }\end{array}$ \\
\hline Wenuy & friend \\
\hline Werken & community spokesperson \\
\hline Wetripantu & $\begin{array}{l}\text { the Mapuche's annual celebration of the repetition of the } \\
\text { earth's cycle around the sun, beginning with the shortest } \\
\text { night in the southern hemisphere on the night of June 20 }\end{array}$ \\
\hline Wigka (sometimes huinca) & $\begin{array}{l}\text { denominates a non-Mapuche person or an outsider to } \\
\text { the Mapuche society in a rather derogatory way }\end{array}$ \\
\hline Wayontamapukeyuaful & international solidarity \\
\hline Yanakona & Traitor \\
\hline Zeuma iyael & $\begin{array}{l}\text { the practice of and knowledge about preparing tradi- } \\
\text { tional Mapuche food, mostly by Mapuche women }\end{array}$ \\
\hline
\end{tabular}




\section{C: List of Personal Interviews}

\begin{tabular}{|c|c|}
\hline Name & Date \\
\hline Alex Mora & November 28, 2015 \\
\hline Alma & $\begin{array}{l}\text { Conversations: } \\
\text { February-April, } 2016\end{array}$ \\
\hline Amanda & $\begin{array}{l}\text { Two interviews: } \\
\text { July 1, } 2016 \\
\text { July 5, } 2016\end{array}$ \\
\hline Amina & November 27, 2015 \\
\hline Andrea Cotrena & June 6, 2017 \\
\hline Cecilia Necul & March 10, 2016 \\
\hline Clarissa & January 22, 2016 \\
\hline Cristián & March 8, 2016 \\
\hline Eva & $\begin{array}{l}\text { Three interviews: } \\
\text { December 1, 2015a } \\
\text { December 1, 2015b } \\
\text { January 29, } 2016\end{array}$ \\
\hline Federico Aguirre & March 2, 2016 \\
\hline Fernando Díaz & March 26, 2016 \\
\hline Cloria Marivil & February 23, 2016 \\
\hline Greta & December 12, 2015 \\
\hline Isabel Cañet & February 24, 2016 \\
\hline Isabell & June 9, 2016 \\
\hline Isidora & June 9, 2016 \\
\hline Jaime Huenchullán & $\begin{array}{l}\text { Two interviews: } \\
\text { March 18, } 2016 \\
\text { March 20, } 2016\end{array}$ \\
\hline Jose Luis Calfucura & $\begin{array}{l}\text { Two interviews: } \\
\text { February 16, 2016a } \\
\text { February 16, 2016b }\end{array}$ \\
\hline Juan Fuenzálida & March 12, 2016 \\
\hline
\end{tabular}




\begin{tabular}{|l|l|}
\hline Karin & January 22, 2016 \\
\hline Kira & February 29, 2016 \\
\hline Llanquiray Painemal & June 16, 2017 \\
\hline Luis and Nadia Painefil & March 10, 2016 \\
\hline Madelaine & December 6, 2015 \\
\hline Maike & June 9, 2016 \\
\hline María Teresa Loncón & March 3, 2016 \\
\hline Matías & March 28, 2016 \\
\hline Mauricio Painefil & March 10, 2016 \\
\hline Mauricio Vergaras & Two interviews: \\
\hline Pastoral Mapuche group discussion & February 25, 2016a \\
\hline Peter & February 24, 2016b \\
\hline Rayen Kvyeh & April 6, 2016 \\
\hline Rike & December 1, 2015 \\
\hline Rubén Sanchez & March 1, 2016 \\
\hline Sabrina & May 27, 2016 \\
\hline Selma and Ramón Necul & March 1, 2016 \\
\hline Sybille & February 4, 2016 \\
\hline The Hague group discussion & March 11, 2016 \\
\hline Verena & June 26, 2016 \\
\hline Vicente Painel & May 5, 2015 \\
\hline Victor Carilaf & December 6, 2015 \\
\hline & Two interviews: \\
\hline February 20, 2016 \\
March 3, 2016 \\
\hline February 23, 2016 \\
\hline
\end{tabular}


